\title{
Fatores influentes para a adesão de alunos a sistema de carona dinâmica em campus universitário
}

\author{
Laize Andréa de Souza Silva ${ }^{1}$, Maurício Oliveira de Andrade ${ }^{2}$, Maria Leonor Alves Maia ${ }^{3}$ \\ ${ }^{1}$ Centro de Tecnologia e Geociências, UFPE, laize.civil.upe@gmail.com \\ ${ }^{2}$ Centro de Tecnologia e Geociências, UFPE, mauandrade@gmail.com \\ ${ }^{3}$ Centro de Tecnologia e Geociências, UFPE, nona@ufpe.br
}

\section{Recebido:}

20 de novembro de 2017

Aceito para publicação:

10 de junho de 2019

Publicado:

31 de agosto de 2019

Editor de área:

Helena Beatriz Cybis

\section{Palavras-chaves:}

Carona;

Sistemas dinâmicos de carona; Comportamento em transportes; Compartilhamento de viagens.

\section{Keywords:}

Carpooling;

Dynamic carpooling systems;

Transport behavior;

Travel sharing.

DOI:10.14295/transportes.v27i2.1521

\begin{abstract}
RESUMO
Este artigo objetiva a investigação dos fatores que influenciam a oferta de carona por alunos do Campus Recife da Universidade Federal de Pernambuco. Investigam-se os critérios relevantes para a adesão a um hipotético sistema dinâmico de caronas. São ainda sugeridas medidas mais eficazes para ampliação do uso dessa alternativa de transporte. $O$ método de análise utilizado foi a Regressão Logística. Os resultados obtidos mostram que fatores mutáveis, inerentes ao arranjo da carona, e psicológicos estão relacionados ao modo dinâmico. Enquanto o único critério fixo que afeta a probabilidade de uso do sistema proposto é o sexo do usuário, seis critérios intrínsecos do próprio sistema apresentam-se significativos: i) perfil do usuário; ii) possibilidade de ser passageiro através do sistema; iii) questões ambientais; iv) maior interação social; v) redução da flexibilidade; e vi) compartilhar viagens com desconhecidos. Conclui-se que o uso da carona pode ser aumentado a partir de sistemas de caronas dinâmicas. Além disso, junto às plataformas de conexão, é importante que sejam adotadas políticas de caráter informativo e educacional.
\end{abstract}

\begin{abstract}
This paper aims to investigate the factors influencing carpooling supply by students from Recife Campus of Federal University of Pernambuco. In addition, some efficient measures for carpooling expansion are identified and suggested. Logistic Regression was the analysis method adopted, using categorical, ordinal and quantitative variables selected based on a review of the literature. The results show that changeable factors that are inherent to the carpooling arrangement and psychological factors are more related to dynamic systems. While the only fixed criterion that affects the probability of using the proposed system is the user's gender, six system intrinsic criteria are significant: i) the user's profile; ii) the possibility of being a passenger through the system; iii) environmental issues; iv) greater social interaction; v) reduction of flexibility; and vi) sharing trips with strangers. It is concluded that carpooling use can be raised by the adoption of dynamic carpooling systems. Furthermore, along the trips connection systems, it is important to assume informative and educational policies in order to encourage changes in travel behaviour and promote carpooling.
\end{abstract}

\section{INTRODUÇÃO}

Reduzir viagens apresenta vantagens ambientais, econômicas e sociais. Carona significa, em geral, compartilhar viagens sem visar lucros. 0 pagamento ao motorista, quando houver, deve apenas cobrir parcialmente os custos operacionais e não objetivar lucros (Chan e Shaheen, 2012). 0 termo carpooling, em inglês, indica a ação (verbo) de compartilhar um automóvel. Este artigo usa o termo quando necessário.

Dentre as vantagens da carona, podem ser destacadas: redução de gastos por meio da divisão do custo da viagem; diminuição dos índices de congestionamentos; menor emissão de gases poluentes; maior interação social; e redução do nível de estresse pelo compartilhamento da 
responsabilidade de dirigir (PENNDOT, 2016). Entretanto, para muitas pessoas a carona não é uma alternativa rotineiramente adotada.

Esse problema é explicado pela vasta gama de variáveis que influenciam na sua adoção, as quais levam a um julgamento que reduz ou desconsidera os benefícios apresentados (Buliung et al., 2009; Tahmasseby, Kattan e Barbour, 2016). Avaliar os fatores que tornam a carona um modo pouco utilizado é fundamental para a formulação de políticas de transporte que estimulem seu uso.

A carona pode ser classificada pelo seu modo de formação, podendo ser casual, programada ou dinâmica. Na primeira categoria, os motoristas aceitam esporadicamente conduzir algum passageiro por motivos diversos, como solidariedade ou divisão de despesas. Por outro lado, a carona programada faz uso de ferramentas computacionais de conexão entre passageiros e motoristas (Waerden, Lem e Schaefer, 2015; Bruglieri et al., 2012).

0 modo programado amplia a possibilidade de realização de caronas, tendo em vista que possibilita a interação entre pessoas desconhecidas que possuem interesses de viagem similares. Contudo, essa forma traz a desvantagem da necessidade de planejar a viagem com antecedência, criando uma noção de dependência de outros, conforme Créno (2014).

Finalmente, o modo dinâmico faz uso da tecnologia de smartphones e recursos de geolocalização para conectar passageiros e motoristas sem a necessidade de agendamentos prévios e acordos detalhados (Créno, 2014; Agatz et al., 2012, 2011). Conforme Gargiulo et al. (2015), tais sistemas foram criados intencionando a popularização dos deslocamentos feitos por carona, tornando-os atrativos para o atendimento de viagens urbanas curtas. Contudo, o bom funcionamento desses sistemas para adesão de usuários depende de massa crítica suficiente para equilibrar os interesses da oferta e da demanda (Créno, 2014). Além disso, deve ser confiável e proporcionar segurança para os usuários (Bruglieri et al., 2012).

Para entender as possibilidades de ampliação dessas alternativas é necessário compreender os fatores que influenciam na escolha da carona. Correia e Viegas (2011) mencionam como principais fatores que impactam a adesão a caronas em Lisboa: custos dos deslocamentos, atributos de tempo e características sócio demográficas dos indivíduos. Schwanen e Lucas (2011) destacam que questões comportamentais e psicológicas relacionadas à insegurança, tornam-se barreiras para o compartilhamento de veículos. Variáveis subjetivas que levam pessoas a optarem pelo automóvel, como conforto e privacidade, também podem representar barreiras para as caronas.

Entretanto, algumas estratégias podem encorajar o uso de caronas como uma alternativa de transporte, especialmente quando aplicadas diretamente a públicos específicos. Os campi universitários, como importantes polos geradores de viagens, representam um espaço onde predomina uma maior relação de confiança interpessoal. Neles, os alunos, na maioria jovens, apresentam características que podem facilitar a aceitação de caronas (Delhomme e Gheorghiu, 2016; Abrahamse e Keall, 2012; Buliung et al., 2009).

Um ponto chave para o sucesso da prática da carona é a confiança percebida pelos usuários. Créno (2014) lista três pontos importantes para a criação da confiança e quebra do "medo do desconhecido" em sistemas dinâmicos de carona: i) garantia de segurança nas transações financeiras, quando houver divisão de custos; ii) criação de um "efeito de tribo", com fortalecimento dos laços sociais entre participantes; e iii) disponibilização de um perfil social online dos envolvidos, para redução do sentimento de desconhecimento do outro. 
Contudo, Agatz et al. (2012) chamam atenção para o fato que nem sempre os participantes podem estar dispostos a fornecer informações pessoais. Sendo assim, de modo geral, a forma encontrada para aumentar a confiabilidade nos sistemas de caronas tem sido a integração com sistemas de avaliação (feedbacks dados pelos próprios usuários) ou com mídias sociais (Gargiulo et al., 2015; Shinde e Thombre, 2015; Agatz et al., 2012).

Na problematização da aceitação da carona, questões sociais e comportamentais devem ser observadas em conjunto para impulsionar a formação de um sistema confiável. Sendo assim, é necessário avaliar os assuntos comportamentais envolvidos. Trabalhos empíricos de comportamento em transportes sugerem que intervir em atitudes pode resultar em mudanças de comportamento relacionadas à escolha modal (Wang e Chen, 2012).

Com base nisso, esta pesquisa tem como bases o conceito dos sistemas dinâmicos de carona, o público universitário como potencial usuário desses sistemas e a necessidade de entender questões comportamentais que dizem respeito à escolha dessa alternativa. A pesquisa busca identificar e avaliar os fatores que influenciam positiva ou negativamente a adesão de alunos universitários ao uso de caronas dinâmicas.

Neste artigo é examinado o caso do Campus Recife da Universidade Federal de Pernambuco, situado a cerca de 12,5 km do centro da cidade, com aproximadamente 35.000 frequentadores por dia. Estudos de Monteiro, Santos e Meira (2015) apontam que 54\% das viagens são realizadas em transporte público, 26,3\% em automóveis e apenas $8 \%$ das pessoas utilizam caronas.

Nesse cenário, busca-se responder em que medida um sistema dinâmico para adesão de usuários, implantado em campi universitários, é atrativo para estudantes que possuem carros. Adicionalmente, objetiva-se apontar estratégias de políticas públicas com potencial de eficácia para incentivo ao seu uso.

\section{REVISÃO DA LITERATURA: CARONA E COMPORTAMENTO}

O conceito da economia do compartilhamento surge no final da década de 1970 (Felson e Spaeth, 1978). No século XXI, com as novas tecnologias da informação, uma nova geração de consumidores fundamentada no uso compartilhado, ao invés de na propriedade de bens, tem impulsionado o funcionamento desse modelo microeconômico (Posen, 2015). Carona como compartilhamento de viagens em veículos privados pode ser considerada parte dessa tendência (Silva; Andrade e Maia, 2018).

Atualmente, tendo em vista que já existe tecnologia suficiente, há uma vasta gama de sistemas que buscam conectar interessados no compartilhamento de viagens (Correia e Viegas, 2011). Assim, um sistema de caronas faz uso de algoritmos para tratar os dados socioeconômicos e locacionais fornecidos pelos cadastrados e realizar a conexão entre agentes. Gardner e Abraham (2008) afirmam que estratégias psicológicas voltadas para atitudes são mais aceitáveis e baratas do que mudanças em infraestruturas. Em meta-análise com 23 bases de dados, os autores constataram que a teoria mais popular aplicada à escolha modal é a Teoria do Comportamento Planejado (TCP) (Ajzen, 1991). Apesar disso, a avaliação das motivações psicológicas é de extrema importância, uma vez que podem se sobrepor aos fatores racionais de julgamento (Neoh, Chipulu e Marshall, 2015).

Estudos sobre o tema são realizados desde quando o carpooling passou a ser visto como uma alternativa de deslocamento benéfica para a mobilidade urbana nas décadas de 1970 e 1980. 
Bonsall, Spencer e Tang (1984), por exemplo, concluíram que a distância percorrida, horários regulares de trabalho, o gênero e a idade dos participantes da carona, bem como o número de habitantes no domicílio são fatores que influenciam a decisão de carpooling.

Embora mais de três décadas tenham passado desde este estudo, algumas tendências se repetem de acordo com pesquisas atuais. A fim de identificar os motivos que encorajam pessoas ao carpooling, Neoh, Chipulu e Marshall (2015) estimaram a influência de 24 fatores de decisão com base em 22 estudos. Eles verificaram que as variáveis de maior impacto são: "sexo", "número de residentes no domicílio", "reserva de vagas de estacionamento", "disponibilidade de faixas exclusivas para veículos com alta ocupação, ou HOV (High Occupancy Vehicles) lanes", "número de empregados", “custos de viagem" e "horário regular de trabalho".

Dentre os fatores sócio demográficos, o sexo é apontado em diversas pesquisas como o principal fator influente. Geralmente, as mulheres tendem mais ao uso desse modo. (Delhomme e Gheorghiu, 2016; Buliung et al. 2009; Li et al., 2008). Apesar dos estudos citados focarem a população em geral, esses resultados se repetem quando a análise é restrita a populações universitárias (Tahmasseby, Kattan e Barbour, 2016; Tezcan, 2016). Contudo, o maior uso pelo sexo feminino não pode ser considerado um fato incontroverso, uma vez que outros estudos apontam resultados divergentes (Correia e Viegas, 2011; Ciari e Axhausen, 2011). Quando as mulheres estão menos inclinadas a carpooling pode existir uma associação à insegurança, que afeta mais a esse grupo.

Uma segunda variável demográfica frequentemente analisada é a idade. Embora muitos estudos concluam que os mais jovens estão mais propensos a aceitar o uso da carona (Delhomme e Gheorghiu, 2016; Abrahamse e Keall, 2012; Buliung et al., 2009;), esse critério pode não apresentar impactos expressivos em outras populações estudadas (Cools et al., 2013). A redução de custos se enquadra na percepção de benefício próprio advindo da oferta da carona. Nesse sentido, vários estudos mostram que o fator econômico influencia positivamente (Cools et al., 2013; Correia e Viegas, 2011; Waerden; Lem e Schaefer, 2015). Entretanto, pesquisa realizada por Tahmasseby, Kattan e Barbour (2016) com estudantes da Universidade de Calgary, conclui que esse grupo se apresenta mais motivado devido a questões ambientais. Esse resultado pode ser justificado pelo nível de educação ou por ser essa uma tendência nas gerações mais jovens.

Justificando o desinteresse pela carona, Li et al. (2008) apontam como motivos: a dificuldade de encontrar pessoas com horários e rotas compatíveis; a dificuldade em realizar viagens encadeadas; e a necessidade do uso do veículo durante o dia. Já Delhomme e Gheorghiu (2016) identificam os não adeptos como pessoas menos preocupadas com problemas ambientais, que consideram mais o conforto dos automóveis e percebem mais desvantagens no uso do transporte público.

Em uma pesquisa realizada em Wellington - Nova Zelândia, Abrahamse e Keall (2012) identificam os seguintes problemas percebidos em usuários um programa de caronas: a dependência de outras pessoas, a baixa flexibilidade, a não disponibilidade do automóvel em situações de emergência e as dificuldades de arranjos em relação à divisão de custos. Adicionalmente, Waerden, Lem e Schaefer (2015), em pesquisa na Holanda com motoristas não familiarizados com caronas, concluem que quanto maior o tempo de viagem ao ponto de encontro e redução de flexibilidade de horários de chegada e partida, menor é o interesse em carpooling. 
Percebe-se na literatura consultada, que a redução da flexibilidade devido a carpooling configura uma barreira ao uso dessa alternativa de transporte. Acredita-se, portanto, que sistemas de carona dinâmica facilitados por algoritmos de conexão, que não imponham grandes desvios de rota e tempos de espera para passageiros e motoristas, tendem a amenizar esse problema de perda da flexibilidade.

\section{METODOLOGIA}

Para responder à questão sobre os fatores que influenciam a decisão de motoristas estudantes universitários de participarem como ofertantes em um sistema de caronas dinâmico foi realizada pesquisa de campo com aplicação de 470 questionários distribuídos proporcionalmente à quantidade de discentes dos centros acadêmicos do campus, entre junho e setembro de 2016, para obtenção de amostra representativa com 95\% de confiança estatística e 5\% de erro considerando-se uma população infinita (amostra mínima de 384). Os respondentes foram selecionados aleatoriamente nos centros acadêmicos, dentre estudantes que utilizavam o carro ao menos uma vez na semana no acesso à universidade.

0 questionário aplicado foi dividido em duas seções: 1) dados sócio demográficos e situacionais fixos; e 2) impressões sobre a possibilidade de uso de um sistema dinâmico de caronas. Na Seção 2, devido à provável falta de familiaridade dos respondentes com o tema, disponibilizou-se um breve texto explicativo sobre sistemas dinâmicos de carona. A resposta à questão do interesse em participar de um sistema de carona dinâmico direcionado à população de sua universidade originou a variável dependente dicotômica "carona" ( $\operatorname{sim}=1$ e não $=0$ ).

Dada a revisão da literatura, foi levantada uma série de variáveis influentes sobre a decisão de carpooling (Chen; Zahiri e Zhang, 2017; Cools et al., 2013; Correia e Viegas, 2011; Delhomme e Gheorghiu, 2016; Neoh e Chipulu; Marshall, 2015; Tezcan, 2016; Waerden; Lem e Schaefer, 2015; Wang e Chen, 2012; Zhou, 2012).

Os fatores foram categorizados em cinco dimensões: demográfica (e.g. renda, sexo, nível de escolaridade); de julgamento, referente à como um indivíduo avalia as vantagens e desvantagens de carpooling (e.g. redução de custos e perda de privacidade); de intervenção, relacionada à interferência de políticas de transportes para incentivo da carona (e.g. implantação de faixas exclusivas de alta ocupação); situacional, que é dependente do ambiente e modo de viagem (e.g. custo do transporte, tempo de viagem); e psicológica, ligada às teorias psicológicas que estão por trás da decisão de carpooling (e.g. acesso a um breve perfil com informações sobre os usuários do sistema, preferência por realizar a viagem sozinho). Na Tabela 1 são listadas as variáveis adotadas para teste do modelo.

Os dados coletados foram analisados por meio do método de regressão logística múltipla, a fim de explicitar as variáveis mais influentes sobre a decisão de um estudante universitário participar de um sistema dinâmico de caronas, além de se obter um modelo previsor da probabilidade de uso desse modelo. A regressão logística é um modelo linear generalizado que possui variável dependente associada a variável aleatória Bernoulli. Esse padrão é o mesmo identificado pela variável "carona" (interesse em participar de um sistema de carona dinâmico). 0 modelo de regressão logística múltipla é descrito pela Equação 1 , tendo $x=\left(x_{1}, \ldots, x_{p}\right)$, onde $p$ é número de previsores:

$$
\operatorname{Logit}[\pi(x)]=\alpha+\beta 1 \times 1+\beta 2 \times 2+\ldots+\beta p x p
$$


Tabela 1: Dimensões de análise e descrição das variáveis.

\begin{tabular}{|c|c|c|}
\hline Dimensões & Variável Codificada & Descrição \\
\hline \multirow{5}{*}{ Fatores demográficos } & Idade & Idade, em anos \\
\hline & Feminino & 0 se masculino e 1 se feminino \\
\hline & Família & Número de pessoas no domicílio \\
\hline & Grau & Nível de escolaridade: 0 se graduação e 1 se pós-graduação \\
\hline & Renda & $\begin{array}{l}\text { Nível de renda média familiar: } 1 \text { se até } 2 \text { salários mínimos; } 2 \text { se entre } 2 \text { e } 5 \\
\text { salários mínimos; } 3 \text { se entre } 5 \text { e } 10 \text { salários mínimos; } 4 \text { se entre } 10 \text { e } 20 \text { salários } \\
\text { mínimos; e } 5 \text { se maior do que } 20 \text { salários mínimos }\end{array}$ \\
\hline \multirow{6}{*}{ Fatores situacionais } & Dias & Número de dias na semana que dirige ao campus \\
\hline & DistMédia & Distância média entre origem-destino, em quilômetros \\
\hline & Dist & $\begin{array}{l}\text { Aumento da distância: } 0 \text { se não apresenta influência; } 1 \text { se é um motivador; e } 2 \\
\text { se é um desmotivador }\end{array}$ \\
\hline & Tempo & $\begin{array}{l}\text { Aumento do tempo: } 0 \text { se não apresenta influência; } 1 \text { se é um motivador; e } 2 \text { se } \\
\text { é um desmotivador }\end{array}$ \\
\hline & CaronaPax & $\begin{array}{l}\text { Uso de carona como passageiro tendo como origem e/ou destino o campus } \\
\text { universitário: } 0 \text { se não utiliza e } 1 \text { se utiliza carona como passageiro }\end{array}$ \\
\hline & CarroPartilhado & 0 se o carro que usa é próprio e 1 se usa carro partilhado \\
\hline \multirow{4}{*}{ Fatores de julgamento } & DivCusto & $\begin{array}{l}\text { Divisão de custos de combustivel: } 0 \text { se não apresenta influência; } 1 \text { se é um } \\
\text { motivador; e } 2 \text { se é um desmotivador }\end{array}$ \\
\hline & Flexibilidade & $\begin{array}{l}\text { Redução de flexibilidade quanto a rotas e horários: } 0 \text { se não apresenta } \\
\text { influência; } 1 \text { se é um motivador; e } 2 \text { se é um desmotivador }\end{array}$ \\
\hline & Privacidade & $\begin{array}{l}\text { Perda de privacidade: } 0 \text { se não apresenta influência; } 1 \text { se é um motivador; e } 2 \text { se } \\
\text { é um desmotivador }\end{array}$ \\
\hline & Ambiente & $\begin{array}{l}\text { Questões de sustentabilidade: } 0 \text { se não apresenta influência; } 1 \text { se é um } \\
\text { motivador; e } 2 \text { se é um desmotivador }\end{array}$ \\
\hline \multirow{2}{*}{$\begin{array}{l}\text { Fatores de } \\
\text { intervenção }\end{array}$} & Estacionamento & $\begin{array}{l}\text { Reserva de vagas para veículos com dois ou mais ocupantes (participantes do } \\
\text { sistema): } 0 \text { se não apresenta influência; } 1 \text { se é um motivador; e } 2 \text { se é um } \\
\text { desmotivador }\end{array}$ \\
\hline & HOV & $\begin{array}{l}\text { Permissão para circular em faixas exclusivas (HOV) se o veículo estiver com três } \\
\text { ou mais ocupantes: } 0 \text { se não apresenta influência; } 1 \text { se é um motivador; e } 2 \text { se é } \\
\text { um desmotivador }\end{array}$ \\
\hline \multirow{4}{*}{ Fatores psicológico } & IntSocial & $\begin{array}{l}\text { Maior integração social: } 0 \text { se não apresenta influência; } 1 \text { se é um motivador; e } 2 \\
\text { se é um desmotivador }\end{array}$ \\
\hline & Desconhecido & $\begin{array}{l}\text { Compartilhar viagens com desconhecidos: } 0 \text { se não apresenta influência; } 1 \text { se é } \\
\text { um motivador; e } 2 \text { se é um desmotivador }\end{array}$ \\
\hline & Pax & $\begin{array}{l}\text { Possibilidade de utilizar o sistema como passageiro: } 0 \text { se não apresenta } \\
\text { influência; } 1 \text { se é um motivador; e } 2 \text { se é um desmotivador }\end{array}$ \\
\hline & Perfil & $\begin{array}{l}\text { Acesso a um breve perfil dos usuários: } 0 \text { se não apresenta influência; } 1 \text { se é um } \\
\text { motivador; e } 2 \text { se é um desmotivador }\end{array}$ \\
\hline
\end{tabular}

Antes da aplicação do método, foram realizados testes de associação entre variáveis para melhor ajuste do modelo para evitar variáveis correlacionadas. Optou-se pelo método de associação o Rho de Spearman ( $\rho$ ), entre as variáveis ordinais independentes.

As variáveis categóricas são transformadas em variáveis dummy, de modo que xi = 1 para observações registradas na categoria i e xi $=0$, caso não sejam registradas observações. 0 valor de i varia de 1 até o número de categorias (i) menos 1 . Portanto, todas variáveis categóricas de três níveis são tratadas de modo que: $\mathrm{x} 2=1$ se o fator é um motivador; 0 em outro caso; $\mathrm{x} 3=1$ se o fator é um desmotivador; 0 em outro caso; em situações nas quais x $2=0$ e x3 = 0 , admitese que o fator não apresenta influência sobre a decisão de dar carona casualmente e/ou participar de um sistema de caronas.

\section{RESULTADOS E ANÁLISES}

Foram obtidas 465 respostas válidas. Todos os respondentes são alunos do Campus e têm disponibilidade de carro para dirigir até a universidade. A amostra pesquisada apresenta em sua maioria alunos de graduação $(83,4 \%)$, que não costumam dar caronas $(67,8 \%)$, com idade 
entre 18 e 28 anos (92,3\%), que percorrem até $15 \mathrm{~km}$ até o Campus (79,8\%), com renda familiar acima de cinco salários mínimos $(71,4 \%)$ e que dirigem ao campus todos os dias da semana (52,0\%). Informações mais detalhadas são mostradas na Tabela 2.

Tabela 2: Caracterização da amostra

\begin{tabular}{|c|c|c|c|c|c|c|c|}
\hline \multirow{2}{*}{ Variável } & \multirow{2}{*}{ Categoria } & \multicolumn{2}{|c|}{ Frequência } & \multirow{2}{*}{ Variável } & \multirow{2}{*}{ Categoria } & \multicolumn{2}{|c|}{ Frequência } \\
\hline & & $N$ & $\%$ & & & $N$ & $\%$ \\
\hline \multirow[t]{2}{*}{ Grau } & Graduação & 388 & $83,40 \%$ & \multirow[t]{8}{*}{ Família } & 1 & 15 & $3,23 \%$ \\
\hline & Pós-graduação & 77 & $16,60 \%$ & & 2 & 43 & $9,25 \%$ \\
\hline \multirow[t]{2}{*}{ Sexo } & Feminino & 208 & $44,70 \%$ & & 3 & 108 & $23,23 \%$ \\
\hline & Masculino & 257 & $55,30 \%$ & & 4 & 131 & $28,17 \%$ \\
\hline \multirow[t]{2}{*}{ Carro } & Não & 252 & $54,20 \%$ & & 5 & 107 & $23,01 \%$ \\
\hline & Sim & 213 & $45,80 \%$ & & 6 & 38 & $8,17 \%$ \\
\hline \multirow[t]{2}{*}{ CaronaPax } & Não & 305 & $67,80 \%$ & & 7 & 20 & $4,30 \%$ \\
\hline & Sim & 145 & $32,20 \%$ & & 8 & 3 & $0,65 \%$ \\
\hline \multirow[t]{4}{*}{ Idade } & Entre 18 e 28 & 429 & $92,26 \%$ & \multirow[t]{6}{*}{ Renda } & 1 a 2 SM & 16 & $3,40 \%$ \\
\hline & Entre 29 e 39 & 31 & $6,67 \%$ & & $>2$ a $5 \mathrm{SM}$ & 117 & $25,20 \%$ \\
\hline & Entre 40 e 51 & 4 & $0,86 \%$ & & $>5$ a $10 \mathrm{SM}$ & 157 & $33,80 \%$ \\
\hline & Não informado & 1 & $0,22 \%$ & & $>10$ a $20 \mathrm{SM}$ & 112 & $24,10 \%$ \\
\hline \multirow[t]{7}{*}{ DistMédia } & 1 a $5 \mathrm{~km}$ & 68 & $14,62 \%$ & & $>20 \mathrm{SM}$ & 62 & $13,30 \%$ \\
\hline & $>5$ a $10 \mathrm{~km}$ & 165 & $35,48 \%$ & & Não informado & 1 & $0,20 \%$ \\
\hline & $>10$ a $15 \mathrm{~km}$ & 138 & $29,68 \%$ & \multirow[t]{5}{*}{ Dias } & 1 & 34 & $7,31 \%$ \\
\hline & $>15$ a $20 \mathrm{~km}$ & 49 & $10,54 \%$ & & 2 & 53 & $11,40 \%$ \\
\hline & $>20$ a $25 \mathrm{~km}$ & 33 & $7,10 \%$ & & 3 & 73 & $15,70 \%$ \\
\hline & $>25$ a $60 \mathrm{~km}$ & 10 & $2,15 \%$ & & 4 & 63 & $13,55 \%$ \\
\hline & Não informado & 2 & $0,43 \%$ & & 5 & 242 & $52,04 \%$ \\
\hline
\end{tabular}

Dentre os alunos entrevistados, 71,8\% afirmaram ter interesse em participar de um sistema de caronas dinâmico. Foram realizados testes de correlação entre as variáveis a fim de utilizar os previsores mais apropriadamente para o modelo de regressão logística.

As associações entre as variáveis ordinais foram medidas pelo teste de Spearman. As interações mais fortes $\left(r_{s}>0,300\right)$ são mostradas na Tabela 3.

Tabela 3: Associações entre variáveis ordinais.

\begin{tabular}{llll}
\hline Variáveis & & Spearman $\left(\mathbf{r}_{\mathbf{s}}\right)$ & valor- $\boldsymbol{p}$ \\
\hline Estacionamento & HOV & 0,437 & 0,000 \\
\hline \multirow{2}{*}{ Privacidade } & Flexibilidade & 0,377 & 0,000 \\
& Desconhecidos & 0,484 & 0,000 \\
\hline \multirow{2}{*}{ Flexibilidade } & Dist & 0,481 & 0,000 \\
& Tempo & 0,460 & 0,000 \\
\hline Dist & Tempo & 0,662 & 0,000 \\
\hline
\end{tabular}

A Tabela 4 mostra o resultado final da regressão.

Tabela 4: Resultado da regressão logística previsor do interesse da participação de um universitário em um sistema de carona dinâmico

\begin{tabular}{|c|c|c|c|c|c|c|c|c|}
\hline \multirow{2}{*}{ Variáveis } & \multirow[b]{2}{*}{ B } & \multirow[b]{2}{*}{ S.E. } & \multirow{2}{*}{ Wald } & \multirow{2}{*}{$D f$} & \multirow{2}{*}{ valor-p } & \multirow{2}{*}{$\operatorname{Exp}(B)$} & \multicolumn{2}{|c|}{ I.C. de $95 \%$ para $\operatorname{Exp(B)}$} \\
\hline & & & & & & & Inferior & Superior \\
\hline Feminino & $-0,920$ & 0,264 & 12,137 & 1 & $0,000^{*}$ & 0,399 & 0,238 & 0,669 \\
\hline IntSocial & & & 14,095 & 2 & $0,001 *$ & & & \\
\hline IntSocial(1) & 0,801 & 0,271 & 8,759 & 1 & $0,003^{*}$ & 2,227 & 1,311 & 3,785 \\
\hline IntSocial(2) & $-0,942$ & 0,645 & 2,131 & 1 & 0,144 & 0,390 & 0,110 & 1,381 \\
\hline Pax & & & 10,675 & 2 & $0,005^{*}$ & & & \\
\hline $\operatorname{Pax}(1)$ & 0,908 & 0,282 & 10,367 & 1 & $0,001^{*}$ & 2,480 & 1,427 & 4,310 \\
\hline $\operatorname{Pax}(2)$ & 0,272 & 0,671 & 0,164 & 1 & 0,686 & 1,312 & 0,352 & 4,890 \\
\hline Desconhecido & & & 23,836 & 2 & $0,000^{*}$ & & & \\
\hline
\end{tabular}


Tabela 4: Resultado da regressão logística previsor do interesse da participação de um universitário em um sistema de carona dinâmico (continuação).

\begin{tabular}{lllllllll}
\hline \multirow{2}{*}{ Variáveis } & \multirow{2}{*}{ B } & S.E. & Wald & Df & valor-p & Exp(B) & \multicolumn{2}{c}{ I.C. de 95\% para Exp(B) } \\
& & & & & & \multirow{2}{*}{ Inferior } & Superior \\
\hline Desconhecido(1) & 1,308 & 0,945 & 1,917 & 1 & 0,166 & 3,700 & 0,580 & 23,588 \\
Desconhecido(2) & $-1,206$ & 0,288 & 17,519 & 1 & $0,000^{*}$ & 0,299 & 0,170 & 0,527 \\
$\begin{array}{l}\text { Flexibilidade } \\
\text { Flexibilidade(1) }\end{array}$ & $-1,837$ & 0,826 & 4,948 & 2 & $0,007^{*}$ & & & \\
Flexibilidade(2) & $-0,924$ & 0,331 & 7,798 & 1 & $0,026^{* *}$ & 0,159 & 0,032 & 0,804 \\
Perfil & & & 12,320 & 2 & $0,002^{*}$ & 0,397 & 0,207 & 0,759 \\
Perfil(1) & 0,969 & 0,320 & 9,184 & 1 & $0,002^{*}$ & 2,634 & 1,408 & 4,928 \\
Perfil(2) & $-0,361$ & 0,658 & 0,301 & 1 & 0,583 & 0,697 & 0,192 & 2,533 \\
Ambiente & & & 7,096 & 2 & $0,029^{* *}$ & & & \\
Ambiente(1) & 0,702 & 0,297 & 5,606 & 1 & $0,018^{* *}$ & 2,018 & 1,129 & 3,610 \\
Ambiente(2) & $-1,098$ & 1,402 & 0,613 & 1 & 0,434 & 0,334 & 0,021 & 5,207 \\
Constante & 0,568 & 0,415 & 1,874 & 1 & 0,171 & 1,764 & & \\
\hline
\end{tabular}

Os fatores que influenciam a participação em um sistema de caronas, de acordo com o modelo final de Regressão Logística obtido, são: "Feminino", “IntSocial”, “Pax", “Desconhecido", “Flexibilidade”, “Perfil” e “Ambiente”. Esses previsores são justamente os que apresentam maior associação com a variável de saída "carona" ("Feminino" dentre os dados fixos e as demais dentre os fatores intrínsecos dos sistemas de carona), com exceção da variável explicativa "Flexibilidade", que apresenta V de Cramér inferior a "Privacidade". Contudo, sendo essa última correlacionada com a primeira e com "Desconhecido", a redução de flexibilidade quanto a rotas e horários se apresentou mais adequada ao modelo do que a queda de privacidade.

A questão da divisão de custo não faz parte do modelo final. Sendo assim, os respondentes se mostram dispostos a usar carona independente da renda, como igualmente concluído por Tezcan (2016). Desse modo, seria mais atrativo propor uma remuneração através de bônus/descontos para utilização de serviços de empresas patrocinadoras do sistema, do que a divisão de custo propriamente dita.

Outros dos dois fatores também correlacionados com a variável "carona", apesar da associação ser considerada fraca, são a adoção de faixas de alta ocupação ("HOV") e reservas de vagas de estacionamento ("Estacionamento"). Assim como avaliado para o critério custo, o uso dessas variáveis elevaria o poder de explicação do modelo (maior $\mathrm{R}^{2}$ de Nagelkerke). Porém, associados a outros critérios, essas variáveis apresentaram-se não significativas.

A estatística de aderência de Hosmer e Lemeshow avalia o ajuste dos dados no modelo, testando a hipótese de que os dados observados são significantemente diferentes do previsto. Desse modo, é importante que esse teste seja não significativo. 0 teste de Hosmer e Lemeshow apresentou valor- $\mathrm{p}=0,261\left(\mathrm{df}=8, \chi^{2}=8,890\right.$, sendo o $\chi^{2}$ crítico $=11,07$ para valor- $\mathrm{p}=0,05$ ), ou seja, os dados observados não são significativamente diferentes do previsto.

A exatidão de previsão da regressão obtida com as variáveis selecionadas é de $81,5 \%$. As estatísticas-R utilizadas na regressão logística se baseiam na verossimilhança logarítmica e seus valores mostram como a inclusão de variáveis previsoras afeta a aderência do modelo. Portanto, o $\mathrm{R}^{2}$ de Nagelkerke é utilizado, sendo ele mais indicado para modelos de escolha dicotômica (Gujarati e Porter, 2008). $0 \mathrm{R}^{2}$ de Nagelkerke do modelo foi equivalente a 0,407. Esse valor indica que o modelo apresenta grau de aderência relativamente baixo.

Tal problema de aderência corresponde ao elevado número de variáveis que podem ter correlação com carpooling e são difíceis de mensurar devido à subjetividade correlacionada aos 
processos de tomada de decisão. Entretanto, de acordo com os outros critérios analisados, a regressão em questão se mostra como um bom modelo previsor da probabilidade de uso de um sistema de caronas dinâmico.

0 valor-p das variáveis apresentadas sem coeficiente (categorias de referência) indica a significância global da variável categórica transformada em dummy.

Dentre as variáveis inerentes a um sistema dinâmico de caronas, todas, com exceção de "Flexibilidade", apresentam em um dos binários uma inconsistência em relação aos limites inferiores e superiores do exp ( $\beta$ ). Portanto, através desta análise é possível confirmar se determinado fator atua como um motivador ou desmotivador. De acordo com o modelo, a variável "Flexibilidade" é significativa a 5\% e a 1\% quando categorizada como um critério motivador e desmotivador, respectivamente. Contudo, quando tratada como desmotivador, ela tende a reduzir a chance de participação em 0,40 vezes. Por outro lado, na posição de motivador, essa chance é reduzida em 0,16 vezes.

Esses resultados exprimem que, apesar da minimização da flexibilidade poder ser apontada como um fato motivador do uso do sistema, a variável "Flexibilidade" sempre reduz a chance de participação, independente do julgamento, conforme observado na literatura (Abrahamse; Keall, 2012; Waerden; Lem e Schaefer, 2015). Adicionalmente, apenas 2,4\% da amostra estudada considerou esse critério como positivo, enquanto $29,29 \%$ informou que ele não influencia na decisão de fazer parte do sistema, e 68,4\% disse que desmotiva, mostrando que é raro que se julgue esse critério como positivo.

Em resumo, o modelo final da Regressão Logística é dado pela Equação (2).

$\operatorname{logit}[\pi(x)]=$

0,568 - 0,920 Feminino + 0,801 IntSocial(1) - 0,942 IntSocial(2) + 0,908 Pax(1) +

0,272 Pax(2) + 1,308 Desconhecido(1) - 1,206 Desconhecido(2) - 1,837

Flexibilidade(1) - 0,924 Flexibilidade(2) + 0,969 Perfil(1) - 0,361 Perfil(2) + 0,702

Ambiente(1) - 1,098 Ambiente(2)]

Dado o modelo, é mais provável que o sistema seja utilizado por um aluno da UFPE do sexo masculino, que tenha interesse na interação social proporcionada pelo serviço, acredite que a possibilidade de se tornar passageiro é uma vantagem, bem como ter acesso a um breve perfil dos usuários, e que considere questões ambientais. A probabilidade de sucesso do uso do sistema dinâmico de caronas sugerido, considerando que a realização de viagens com desconhecidos e a redução de flexibilidade promovida pela carona são pontos negativos é de aproximadamente $86 \%$.

Em contrapartida, características opostas podem reduzir a probabilidade de participação para até $0,98 \%$. Assim, comprova-se que a variação de fatores psicológicos (interação social, realização viagem como passageiro e acesso um perfil do usuário), bem como o sexo e questões ambientais pode reduzir a probabilidade de uso do sistema em até 85,05\%.

Em análise do modelo, tem-se como facilitadores da entrada no sistema: i) o acesso a um breve perfil dos usuários, que aumenta a chance de alguém participar do sistema em 163\%; ii) a possibilidade de sair da posição de motorista para passageiro, um atrativo que aumenta em $148 \%$ a chance de sucesso do programa; iii) o desejo por interação social durante os deslocamentos, que aumenta em $123 \%$ a chance de participação no sistema de caronas; e iv) as preocupações com questões ambientais, que maximizam a probabilidade de sucesso com chance de participação 102\% maior. 
Por outro lado, são desmotivadores os seguintes fatores: i) realizar viagens com desconhecidos, que reduz em $70 \%$ a probabilidade de sucesso do sistema; ii) o fato de ser mulher, dado que são cerca de $60 \%$ mais resistentes a utilizar o sistema; e iii) a redução de flexibilidade ocasionada pelo modo carona, que minimiza a chance de participação no sistema proposto em $84 \%$ quando considerado um motivador, e em $60 \%$ quando julgado como desmotivador.

Os resultados apontam que os sistemas de carona dinâmicos apresentam caráter subjetivo no que diz respeito à participação de potenciais usuários. Enquanto o único critério fixo influente referente ao sistema proposto é o sexo do usuário, seis outros critérios (des)motivacionais intrínsecos do próprio sistema apresentam peso bastante significativo. Essa tendência converge para as conclusões de Abrahamse e Keall (2012), que mostram em seus estudos que variáveis sócio demográficas explicaram apenas 3\% da variância na frequência em dirigir sozinho para o trabalho, enquanto as crenças especificadas explicaram $23 \%$.

As questões de gênero aparecem de forma bastante relevante nos resultados. Os discentes do sexo feminino apresentam-se mais resistentes ao uso de um sistema de carona dinâmico. Esse resultado diverge das conclusões de diversos estudos que relatam maior participação do sexo feminino em carpooling (Li et al., 2008; Buliung et al., 2009; Delhomme e Gheorghiu, 2016). Contudo, para a população estudada neste artigo, esse comportamento pode estar relacionado ao fato das mulheres tenderem a se sentir mais receosas por serem mais vulneráveis a algum tipo de violência, conforme comentários feitos por algumas entrevistadas.

A variável referente à sustentabilidade da carona também é influente para a adesão de usuários. Esse fator se apresenta como um motivador que eleva a probabilidade de participação em um sistema dinâmico em aproximadamente 102\%, sendo o critério menos importante dentre os motivadores.

\section{DISCUSSÃO DOS RESULTADOS}

Dado que as mulheres são mais receosas em aderir a um sistema dinâmico de caronas, pode-se concluir que o desconhecido está psicologicamente atrelado a questões de falta de segurança, mais especificamente, de violência contra a mulher. Apesar desses resultados, acredita-se que essa desconfiança ao uso da carona dinâmica por mulheres possa ser mitigada. Um sistema dinâmico de adesão direcionado à população universitária poderia oferecer um serviço com grupos de viagem formados apenas por mulheres. Essa medida fortalece a criação do "efeito de tribo" proposto por Créno (2014) ao identificar que o estreitamento dos laços sociais entre os participantes pode evitar a rejeição da carona.

Além dos grupos formados apenas por mulheres, é importante inserir no sistema uma ferramenta de avaliação, através da qual, passageiros e motoristas troquem notas e/ou comentários que apontem o sucesso ou fracasso da parceria. Esse processo visa reduzir o sentimento de desconhecimento do outro, fator que é importante não apenas para as mulheres: como visto na literatura. 0 perigo relacionado à interação com estranhos é um dos primeiros obstáculos à adoção de carona (Tahmasseby, Kattan e Barbour, 2016).

De acordo com o modelo estudado, a possibilidade de enxergar o outro através de um perfil de usuário é o que mais importa na decisão de participar do sistema. Portanto, as avaliações de Créno (2014) sobre a necessidade de disponibilização de um perfil social online dos envolvidos, para minimizar sentimento de desconhecimento do outro, e elevar a confiança e segurança, é confirmada. 
Outra forma de aumentar a sensação de segurança dos usuários é pela inserção no sistema de um perfil não apenas dos usuários, mas também do veículo utilizado pelo ofertante. Relatos feitos durante as entrevistas constataram que as pessoas se sentem mais seguras em relação a realizar viagens com desconhecidos quando têm informações sobre o veículo. Portanto, alguns dados como placa e ano do automóvel podem auxiliar na construção da confiança entre o sistema e seus usuários.

É interessante frisar que, apesar do compartilhamento de viagens com desconhecidos ser um desmotivador, o aumento da interação social foi visto como um ponto positivo. Esse resultado confirma um paradoxo de comportamento: ao mesmo tempo em que as pessoas sentem necessidade de interagir e conhecer novas pessoas têm medo de uma experiência ruim derivada do desconhecimento do outro.

Um dos critérios inerentes dos sistemas que mais incentiva a participação é o fato dos usuários poderem sair da posição de motorista para a de passageiro (fato que aumenta em $148 \%$ a chance de participação). Essa questão está em conformidade com a análise do dilema social, tendo em vista que usufruir do conforto proporcionado pelo automóvel sem a necessidade de utilizar o próprio veículo e não pagar a mais por isso soa como uma ótima opção particular de deslocamento.

Em relação às questões ambientais, o resultado obtido mostra que a população estudada tem certa percepção do impacto do uso do automóvel sobre a emissão de gases poluentes e suas implicações sobre o efeito estufa. Utilizando essa questão para impulsionar a participação em um sistema de caronas, seria interessante inserir no programa uma calculadora de redução da emissão de $\mathrm{CO}_{2}$ proporcionada pela carona. Assim, ao atingir determinado patamar estipulado, o usuário receberia alguma bonificação dada pelos patrocinadores do sistema, tornando seu uso mais atrativo.

As medidas infraestruturais e regulamentares de incentivo à carona levantadas na pesquisa (adoção de faixas HOV e reserva de vagas de estacionamento) não são de grande importância para promoção de carpooling. Tendo-se em vista que HOV lanes para caronas é uma medida de gerenciamento de demanda não popularizada no Brasil e ausente na Região Metropolitana do Recife (RMR), a pouca importância dada a essa medida pode ser justificada.

Apesar das políticas possíveis de serem tomadas para tornar carpooling mais atrativo, alguns fatores não deixam de ser desmotivadores subjetivos e difíceis de serem tratados. 0 desejo de flexibilidade e independência é um ponto negativo para carpooling constatado desde a década de 1980 (Bonsall; Spencer e Tang, 1984). De acordo com as análises realizadas, acredita-se que a formulação de um sistema que garanta a segurança dos participantes e tenha um algoritmo robusto para manter o máximo possível da flexibilidade característica do transporte individual, ou seja, sem elevados desvios de rota e tempos de espera, elevará a popularização dessa alternativa de deslocamento.

A redução de privacidade é um critério extremamente individual que afeta diferentemente cada pessoa. As questões já mencionadas visam minimizar o impacto causado pelo desconhecimento do outro e atuam para quebrar também essa barreira. Conforme Créno e Cahour (2014), a reputação do serviço online e a transparência das informações são de extrema importância. Assim, o desejo de participação não significa continuidade do uso do sistema. É necessário que a utilidade da ferramenta seja monitorada e que os usuários tenham a quem recorrer no caso da quebra de confiança. 
Adicionalmente, deve-se reforçar a conscientização dos custos pessoais e sociais de dirigir sozinho, tendo-se em vista que a população estudada já mostrou apresentar boa percepção dos danos ambientais do transporte individual.

\section{CONCLUSÕES}

Apesar dos objetivos sociais e ambientais, a carona ainda apresenta baixa aceitabilidade quando se trata do compartilhamento de viagens entre desconhecidos. Tratando-se da complexidade do comportamento humano e dos processos de tomada de decisão, a simples existência de um sistema dinâmico para adesão não garante o seu sucesso.

Essa pesquisa buscou identificar entre alunos do Campus Recife da UFPE, que utilizam o automóvel para ir à universidade, quais os fatores são mais influentes sobre a decisão de participar de um sistema de caronas hipotético proposto. Embora os estudantes universitários sejam considerados mais abertos à aceitação de mudanças comportamentais que a escolha do modo carona exige, com base na literatura estudada não foram identificadas diferenciações entre o grupo abordado e outras populações não universitárias.

Esta pesquisa confirma o que a literatura aponta no que diz respeito a grande variabilidade nos fatores que afetam o uso da carona. Esse fato é confirmado pelo baixo poder de explicação do modelo obtido $\left(\mathrm{R}^{2}\right.$ de Nagelkerke de 0,407). Portanto, os critérios influentes sobre carpooling são diferenciados a depender do local, população e contexto analisado. Ademais, ainda que sejam identificados os critérios mais atuantes, é difícil prever o comportamento das pessoas devido à subjetividade correlacionada aos processos de tomada de decisão.

A aplicação de políticas de cunho informativo e educacional que objetivem encorajar mudanças de comportamento e promover carpooling, apresentam-se mais importantes do que interferências em infraestruturas (e.g. implementação de HOV lanes), e medidas econômicas (e.g. implementação de faixas para veículos de alta ocupação em vias pedagidas - HOT high occupancy toll lanes). Portanto, focar nos benefícios para a mobilidade urbana e meio ambiente, pode ser o modo mais eficiente de despertar o interesse dos universitários de participarem de um sistema dinâmico de caronas.

Junto a isso, políticas regulamentares devem ser avaliadas e cuidadosamente implantadas. Atualmente, o Campus Recife - UFPE é ausente de um pacote de medidas que busque a aplicação de estratégias de incentivo do uso de modos não motorizados e a redução do uso do automóvel. Entretanto, tratando qualquer campus universitário como polo gerador de viagem, é importante que seja adotada uma Política Institucional de Mobilidade Sustentável. Desse modo, em uma política mais ampla, estratégias para tornar carpooling mais atrativo constituem uma das medidas que podem ser postas em prática.

Conclui-se que o uso da carona pode ser elevado a partir de sistemas dinâmicos é confirmada. Numericamente, a pesquisa confirmou que aproximadamente $74 \%$ dos entrevistados que declararam não dar carona casualmente têm interesse em participar do sistema proposto. Isso significa que a oferta de carpooling poderia aumentar, uma vez que os apenas $9,5 \%$ das pessoas continuariam não utilizando carona, número que antes era de 36\%.

Os sistemas de caronas aplicados a campi universitários podem ser viáveis, apesar das inúmeras variáveis subjetivas e de difícil identificação relacionadas. Entretanto, é necessário que sejam adotadas medidas de segurança que maximizem a confiança empregada no sistema, tendo em vista que o parceiro de viagem é um desconhecido, e que seja despertado o engajamento socioambiental proveniente de seu uso. 


\section{REFERÊNCIAS}

ABRAHAMSE, W. e KEALL, M. (2012) Effectiveness of a web-based intervention to encourage carpooling to work: A case study of Wellington. New Zealand. Transport Policy, v. 21, p. 45-51. https://doi.org/10.1016/j.tranpol.2012.01.005

AGATZ, N.; ERERA, A.; SAVELSBERGH, M. e WANG, X. (2012) Optimization for dynamic ride-sharing: A review. European Journal of Operational Research, v. 223, n. 2, p. 295-303. https://doi.org/10.1016/j.ejor.2012.05.028

AGATZ, N.; ERERA, A.; SAVELSBERGH, M. e WANG, X. (2011) Dynamic ride-sharing: A simulation study in metro Atlanta. Transportation Research Part B, v. 45, n. 9, p. 1450-1464. https://doi.org/10.1016/j.trb.2011.05.017

AJZEN, I. (1991) The theory of planned behavior. Organizational Behavior and Human Decision Processess, v. 50, n. 2, p. 179211. Disponível em: https://doi.org/10.1016/0749-5978(91)90020-T. BANISTER, D. e MARSHALL, S. (2000) Encouraging transport alternatives. Good practice in reducing travel. The Stationery Office, Norwich. URL: http://worldcat.org/isbn/0117023884.

BONSALL, P. W.; SPENCER, A. H. e TANG, W.S. (1984) What makes a car-sharer? Transportation, v. 12, p. 117-145. https://doi.org/10.1007/BF00167372

BRUGLIERI, M.; Ciccarellib, D.; Colornia, A. e Luè, A. (2012) PoliUniPool: a carpooling system for universities. Social and Behavioral Sciences, v. 20, n. 2011, p. 558-567. https://doi.org/10.1016/j.sbspro.2011.08.062

BULIUNG, R. N.; SOLTYS, K.; HABEL, C. e LANYON, R. (2009) The "Driving" Factors behind Successful Carpool Formation and Use. Transportation, v. 651, n. August, p. 1-17. https://doi.org/10.3141/2118-05

CHEN, X. M.; ZAHIRI, M. e ZHANG, S. (2017) Understanding ridesplitting behavior of on-demand ride services : An ensemble learning approach. Transportation Research Part C, v. 76, p. 51-70. https://doi.org/10.1016/j.trc.2016.12.018

CIARI, F. e AXHAUSEN, K. W. (2011) Choosing carpooling or carsharing as a mode: Swiss stated choice experiments (I). Arbeitsbericht Verkehrs und Raumplanung. Zurique, Suíça.

COOLS, M.; TORMANS, H.; BRIERS, S. e TELLER, J. (2013) Unravelling the determinants of carpool behaviour in Flanders, Belgium: Integration of qualitative and quantitative research. Anais do BIVEC/GIBET Transport Research Day. Hesse, M.; G. Caruso; P. Gerber e F. Viti (eds.). Zelzate: University Press.

CORREIA, G. e VIEGAS, J. M. (2011) Carpooling and carpool clubs: Clarifying concepts and assessing value enhancement possibilities through a Stated Preference web survey in Lisbon, Portugal. Transportation Research Part A: Policy and Practice, v. 45, n. 2, p. 81-90. https://doi.org/10.1016/j.tra.2010.11.001

CRÉNO, L. (2014) Energy Consumption and Autonomous Driving . Anais do 3rd CESA Automotive Electronics Congress. Paris. https://doi.org/10.1007/978-3-319-19818-7

CRÉNO, L. e CAHOUR, B. (2014) Chronicles of Lived Experiences for studying the process of trust building in carpooling. Anais do European Conference on Cognitive Ergonomics. Vienna. https://doi.org/10.1145/2637248.2637255

DELHOMME, P. e GHEORGHIU, A. (2016) Comparing French carpoolers and non-carpoolers: Which factors contribute the most to carpooling? Transportation Research Part D, v. 42, p. 1-15. Acesso em: 07 Jun. 19. https://doi.org/10.1016/j.trd.2015.10.014

FELSON, M. e SPAETH, J. L. (1978) Community Structure and Collaborative Consumption: A Routine Activity Approach. The American Behavioral Scientist, v. 21, n. 4, p. 164. https://doi.org/10.1177/000276427802100411

GARDNER, B. e ABRAHAM, C. (2008) Psychological correlates of car use: A meta-analysis. Transportation Research Part F: Traffic Psychology and Behaviour, v. 11, n. 4, p. 300-311. https://doi.org/10.1016/j.trf.2008.01.004

GARGIULO, E.; GIANNANTONIOA, R.; GUERCIOA, E.; BOREANA, C. e Zenezinib, G. (2015) Dynamic ride sharing service: are users ready to adopt it? Procedia Manufacturing, v. 3, p. 777-784. https://doi.org/10.1016/j.promfg.2015.07.329

GUJARATI, D. N. e PORTER, D. C. (2008) Econometria Básica. 5. ed., Mc Gram Hill. Bookman, Nova York. ISRN: 078.85.8055.051.1.

LI, J.; EMBRY, P.; MATTINGLY, S. P.; SADABADI, K. F.; RASMIDATTA, I. e BURRIS, M. W. (2008) Who Chooses to Carpool and Why? Examination of Texas Carpoolers. Transportation Research Record, v. 2021, p. 110-117. https://doi.org/10.3141/2021-13

NEOH, J. G.; CHIPULU, M. e MARSHALL, A. (2015) What encourages people to carpool? An evaluation of factors with metaanalysis. Transportation, n. 16 September 2015, p. 1-25. https://doi.org/10.1007/s11116-015-9661-7

PENNDOT - Pennsylvania Department of Transportation (2016) PACOMMUTES (Alternative Transportation in Pennsylvania). Disponível em: <http://www.pacommutes.com/ridesharing/car-pooling/>. Acesso em: 16 fev.

POSEN, H. A. (2015) Ridesharing in the Sharing Economy: Should Regulators Impose Uber Regulations on Uber? Iowa Law Review, v. 101, n. 1, p. 405-433. Disponível em: https://ilr.law.uiowa.edu/print/volume-19-issue-1/ridesharing-in-thesharing-economy-should-regulators-impose-ueber-regulations-on-uber/. Acesso em: 07 Jun. 19.

SCHWANEN, T. e LUCAS, K. (2011) Understanding Auto Motives. In: Emerald Group Publishing Limited (eds.) Auto Motives: Understanding Car Use Behaviours. Reino Unido. ISBN: 978-0-85-724233-4. https://doi.org/10.1108/9780857242341001

SHINDE, T. e THOMBRE, B. (2015) An Effective Approach for Solving Carpool Service Problems Using Genetic Algorithm Approach in Cloud Computing. International Journal of Advance Research in Computer Science and Management Studies, v. 3, n. 12, p. 29-33. Disponível em: http://www.ijarcsms.com/docs/paper/volume2/issue11/V2I11-0031.pdf. Acesso em: 07 Jun. 19

SILVA, L. A.; ANDRADE, M. e MAIA, M. L. (2018) How does the ride-hailing systems demand affect individual transport regulation? Research in Transportation Economics, v. 69, p. 600-606. 
TAHMASSEBY, S.; KATTAN, L. e BARBOUR, B. (2016) Propensity to participate in a peer-to-peer social-network-based carpooling system. Journal of Advanced Transportation, v. 50, n. August 2015, p. 240-254. https://doi.org/10.1002/atr.1326

TEZCAN, H. O. (2016) Potential of Carpooling among Unfamiliar Users: Case of Undergraduate Students at Istanbul Technical University. Journal of Urban Planning and Development, v. 142, n. 1, p. 1-11. https://doi.org/10.1061/(ASCE)UP.19435444.0000283

WAERDEN, P. V. D.; LEM, A. e SCHAEFER, W. (2015) Investigation of factors that stimulate car drivers to change from car to carpooling in city center oriented work trips. Transportation Research Procedia, v. 10, n. July, p. 335-344. Acesso em: 07 Jun. 19. https://doi.org/10.1016/j.trpro.2015.09.083

WANG, T. e CHEN, C. (2012) Attitudes, mode switching behavior, and the built environment: A longitudinal study in the Puget Sound Region. Transportation Research Part A: Policy and Practice, v. 46, n. 10, p. 1594-1607. https://doi.org/10.1016/j.tra.2012.08.001

ZHOU, J. (2012) Sustainable commute in a car-dominant city: Factors affecting alternative mode choices among university students. Transportation Research Part A: Policy and Practice, v. 46, n. 7, p. 1013-1029. https://doi.org/10.1016/j.tra.2012.04.001 